\title{
Remarks on relativistic kinetic theory to first order in the gradients
}

\author{
A. L. Garcia-Perciante ${ }^{2}$, A. Sandoval-Villalbazo ${ }^{1}$ \\ ${ }^{1}$ Depto. de Fisica y Matematicas, Universidad Iberoamericana, \\ Prolongacion Paseo de la Reforma 880, \\ Mexico D. F. 01219, Mexico and \\ ${ }^{2}$ Depto. de Matematicas Aplicadas y Sistemas, \\ Universidad Autonoma Metropolitana-Cuajimalpa, \\ Artificios 40 Mexico D.F 01120, Mexico.
}

\begin{abstract}
In this paper we emphasize some conceptual points related to the kinetic foundations of relativistic hydrodynamics. We summarize previous work and focus on the construction of the heat flux from a kinetic theory point of view. A thorough discussion addressing aspects concerning stability, causality and the construction of an appropriate stress-energy tensor is included.
\end{abstract}




\section{INTRODUCTION}

The first solid theory formulated to deal with irreversible processes is due to Lars Onsager [1] [2] and its first version was published almost eighty years ago. Extensions and modifications of his method were made by Casimir [3] and almost simultaneously by Meixner [4], all of which gave rise to what we presently know as linear irreversible thermodynamics (LIT). However, in 1940, before LIT was finally accomplished, C. Eckart published three papers on irreversible thermodynamics [5], the first two more or less along the lines of LIT and the third one dealing with the relativistic irreversible thermodynamics of a simple fluid. Thus arises the question: is this work the correct extension of LIT to relativistic systems? The answer has been subject to continuous debate for over forty years and this is precisely the motivation of this work. It is important to point put that no new results are included in this publication. Following a very much appreciated suggestion from an anonymus referee, this review article has been written as a thorough discussion of some key theoretical aspects that lie beneath recent results that can be found in the literature. Some basic equations are included for the sake of clarity.

It has recently been shown [6] that the source of the generic instabilities of a relativistic fluid [7] is the misuse of the constitutive equation for the heat flow first proposed by Eckart in 1940 [5]. In that work it is clearly shown how the acceleration term in the expression

$$
J_{[Q]}^{\nu}=-\kappa h_{\mu}^{\nu}\left(T^{, \mu}+\frac{T}{c^{2}} \dot{u}^{\mu}\right)
$$

is the one that leads to the exponential growth of fluctuations around the equilibrium state in the linearized system of relativistic transport equations. Here $J_{[Q]}^{\nu}$ is the heat flux, $\kappa$ a relativistic thermal conductivity, $h^{\mu \nu}$ is the usual spatial projector defined as $h^{\mu \nu}=g^{\mu \nu}+\frac{u^{\mu} u^{\nu}}{c^{2}}$ for a $(+++-)$ signature, $T$ is the local temperature, $c$ the speed 
of light and $u^{\mu}$ the hydrodynamic four velocity. The covariant derivative is denoted by a semicolon, the ordinary one as a comma and a dot implies a total proper-time derivative such that $\dot{a}^{\nu}=u^{\mu} a_{; \mu}^{\nu}$. In this work we point out two major drawbacks of this constitutive equation and thus proceed to address the matter from the kinetic theory point of view. We repeat some key equations leading to the constitutive relation obtained from the relativistic Boltzmann equation as well as the ones needed in order to address both the violation of the linear regression assumption and the causality of the first order (in the gradients) theory.

To accomplish this task in Section II we start by recalling the basic elements of Onsager's theory in order to clearly expose the shortcomings introduced in first order

theories by Eq. (11). In Section III we outline the calculation of Ref. [8] where a constitutive equation for the heat flux is derived by solving the Boltzmann kinetic equation for a relativistic ideal gas in a BGK-like approximation. In Section IV we briefly summarize the key steps and arguments favoring the kinetic theory result and showing its consistency with linear irreversible thermodynamics. Finally, the key aspects of the work are summarized and discussed in Section V.

\section{GENERIC INSTABILITIES AND ONSAGER'S HYPOTHESIS}

The constitutive equation (11), arises from phenomenologically enforcing the second law of thermodynamics to a system in which the stress-energy tensor includes relativistic corrections terms proportional to the heat flux. Such tensor is obtained as the most general decomposition of a second rank tensor in this framework. This procedure is the standard one in non-equilibrium thermodynamics, however it leads to a consitutive equation which is at odds with its basic tenets in two senses. First, according to this theory, fluxes must be coupled to the forces given by the gradients of the intensive variables. This requirement is clearly not met by the second 
term in Eq.(11), the acceleration is neither an independent variable nor a thermodynamic force. The second problem, as mentioned above, is the fact that it leads to a violation of the linear regression assumption, a behavior usually referred to as "generic intability". As was pointed out in Ref. [6], Onsager's hypothesis states that spontaneous fluctuations of the state variables around the equilibrium state, whose origin is purely microscopic, should relax following the linearized equations for such variables. Thus, the exponential growth obtained by Hiscock and Limdblom is really a violation of this assumption which is key in the construction of irreversible thermodynamics and in particular in the proof of Onsager's reciprocity relations. Indeed, the theory of irreversible processes developed by Onsager seeks the establishment of a connection between these processes and the spontaneous fluctuations that due to their microscopic nature appear in the thermodynamic variables of equilibrium systems. To do so he used the local equilibrium assumption (LEA) [9] [10] by assuming the thermodynamic state of a system to be determined through a set of extensive variables $\left(\alpha_{1}, \alpha_{2}, \ldots, \alpha_{n}\right)=\vec{\alpha}$ (energy, volume, particle number, etc) with which all thermodynamic variables, in particular the entropy $S$, may be defined. These $\alpha^{\prime} s$ are further redefined so that they vanish in the equilibrium state, $S(\vec{\alpha})_{E q}=S(\overrightarrow{0})=S_{0}$ and thus, the thermodynamic forces acting on the system to seek equilibrium are:

$$
X_{i}=\left(\frac{\partial S}{\partial \alpha_{i}}\right)_{j \neq i}
$$

while the fluxes are simply the time derivatives of the $\alpha^{\prime} s$. The essential assumption in Onsager's formulation is that the fluxes are linearly related to the forces $X_{i}$ so that

$$
\frac{d \alpha_{i}}{d t}=\sum_{j=1}^{n} L_{i j} X_{j}
$$

Further, to determine the force $X_{j}$ it is assumed that $S(\vec{\alpha})$ can be expanded in a Taylor series around equilibrium. Since the linear term vanishes and defining 
$\Delta S=S(\vec{\alpha})-S_{0}$ one gets that

$$
\Delta S=-\frac{1}{2} \sum_{j=1}^{n} \sum_{i=1}^{n} g_{i j} \alpha_{i} \alpha_{j}
$$

where the $g_{i j}$ 's are equilibrium properties. Using Eqs. (2) and (44) it follows immediately that the linear laws take the form:

$$
\sum_{j=1}^{n}\left(R_{i j} \frac{d \alpha_{j}}{d t}+g_{i j} \alpha_{j}\right)=0 \quad i=1, . ., n
$$

where $R_{i j}=\left(L_{i j}\right)^{-1}$. Assuming that the spontaneous fluctuations around the equilibrium state of any system obey the same equations as the macroscopic variables, e.g. Eq. (5) , using the methods of statistical mechanics and the fact that the microscopic equations of motion are invariant under time inversions (microscopic reversibility), Onsager was able to show that the matrix $L_{i j}$, the transport coefficients matrix, is symmetric

$$
L_{i j}=L_{j i}
$$

The proof of this statement may be found in any good textbook in statistical mechanics [11]-[14], or in Refs. [1]-[3]. What is of outmost importance is to understand that once we admit the validity of Onsager's Reciprocity Theorem, Eq. (6) , we are implicitly admitting the linear regression of the fluctuations hypothesis, which has been thoroughly corroborated by experiments in colloid systems [15] [16], light scattering of fluids [17] and other systems [18].

However, Casimir [3] and J. Meixner [4] independently pointed out that many irreversible processes did not fit into Onsager's formulation, in particular the cases where the state variables are field variables, such as in heat and electrical conduction. In such cases the state variables satisfy conservation equations and the forces are given by gradients of intensive variables instead of defined as in Eq. (2). Two major changes appear: Eq. (4) is substituted by the entropy balance equation

$$
\rho \frac{d s}{d t}+\operatorname{div} \vec{J}_{s}=\sigma
$$


which results strictly from the validity of the LEA and the conservation equations. In Eq. (7) $\rho$ is the mass density, $\sigma$ is the entropy production and $\vec{J}_{s}$ is the entropy flux, which is a measure of the entropy flowing through the boundaries of the system. Secondly, for an isotropic system $\sigma$ turns out to be a sum of products of forces and fluxes of the same tensorial rank. Thus, the linear relationship in Eq. (3) is consistent with $\sigma>0$ but now the fluxes are no longer the time derivatives of the field variables but the currents. Physically, $\sigma$ is just the local version of what Clausius in his last paper on thermodynamics called the "uncompensated heat", a measure of the heat generated by the dissipative effects occurring in a process taking place in the system [19]. Following the linear relation hypothesis, the entropy production turns out to be a quadratic form in terms of the forces, usually written as

$$
\sigma=\sum_{i=1}^{n} \sum_{k=1}^{n} L_{i k} X_{i} X_{k}
$$

such that, if the transport coefficients $L_{i k}$ are positive, then $\sigma>0$.

Onsager's reciprocity theorem, stated in Eq. (6) , is kept as a potulate in the extensions of his theory that constitute nowadays LIT formalisms. This means that one is ultimately also accepting the linear regression of fluctuations hypothesis. To summarize this section, our objection to the use of Eq. (1) comes from the fact that it is inconsistent with classical irreversible thermodynamics in two senses: it does not have the structure given by Eq. (8) and it leads to a violation of the linear regression of fluctuations hypothesis. The proof of this last statement follows directly from the fluctuaction analysis using standard hydrodynamics techniques. The full calculation is shown in Ref. [6]. 


\section{THE KINETIC THEORY APPROACH}

The contradictions that arise from coupling the hydrodymamic acceleration, or any type of term that is not a gradient of a state variable, with the heat flux have been clearly stated in the previous section. Since this coupling seems to arise from following a phenomenological treatment, one is naturally lead to the question of whether such a relation is predicted by kinetic theory which, as in the classical case provides a microscopic framework for the establishment of constitutive equations from basic principles. The procedure is indeed well-known in the non-relativistic case through the use of Boltzmann's equation for a dilute gas. In the relativistic case, as long as the detailed expressions for the transport coefficients are not required, one can accomplish this task by using the simplified form of the relativistic Boltzmann equation known as Marle's equation [20]. This equation reads,

$$
v^{\alpha} f_{, \alpha}=-\frac{f-f^{(0)}}{\tau}
$$

where $v^{\alpha}$ is the molecular four-velocity, $f$ is the single particle distribution function and $f^{(0)}$ the equilibrium distribution, i. e. the solution to the homogeneous Boltzmann equation. In Eq. (9) $\tau^{-1}$ is a parameter which contains the information arising form the collisions between the particles. However, as mentioned above, this information is not required for our purposes. Such details are required in order to calculate transport coefficientes while the structure, in the sense of the dependence with the state variables, of the dissipative fluxes can be assesed without them.

The solution to Eq.(9) can be obtained by the standard methods of kinetic theory, which have been shown to be valid also in the relativistic case in Ref. [21]. Here we only outline the procedure since the full calculation has been shown elsewhere [8]. In order to obtain the solution to first order in the gradients one uses the ChapmanEnskog method in which the distribution function is proposed as 


$$
f=f^{(0)}(1+\phi)
$$

The first term corresponds to the Euler relativistic regime and the second one, where $\phi$ is the first order correction in the Knudsen parameter, to the Navier-Stokes level. The equilibrium distribution function in Eqs. (9) and (10) is a relativistic Maxwellian which, in the non-degenerate case, is given by [20, 22]

$$
f^{(0)}=\frac{n}{4 \pi c^{3} z \mathcal{K}_{2}\left(\frac{1}{z}\right)} e^{\frac{u^{\beta} v_{\beta}}{z c^{2}}} .
$$

where $m$ is the rest mass of the particles, $z=\frac{k T}{m c^{2}}$ the usual relativistic parameter and $\mathcal{K}_{n}$ the modified Bessel function of the $\mathrm{n}$-th kind. Subtitution of this hypothesis in Eq. (9) leads to

$$
\phi=-\tau v^{\alpha}\left(\frac{\partial f^{(0)}}{\partial n} n_{, \alpha}+\frac{\partial f^{(0)}}{\partial T} T_{, \alpha}+\frac{\partial f^{(0)}}{\partial u^{\beta}} u_{; \alpha}^{\beta}\right)
$$

The derivatives can be readily calulated and subtituted. The Euler (previous order) equations are used to write the time derivatives, appearing in the sum when $\alpha=4$, in terms of the spatial gradients. Notice that this procedure will naturally lead to a coupling of the dissipative fluxes, calculated as moments of $\phi$, with the thermodynamic forces. The solution, in the fluid's comoving frame can be written as

$$
\begin{aligned}
f^{(0)} \phi= & -\tau v^{\ell} f^{(0)}\left[\frac{n_{, \ell}}{n}+\frac{T_{, \ell}}{T}\left(-1+\frac{\gamma}{z}-\frac{\mathcal{K}_{1}\left(\frac{1}{z}\right)}{2 z \mathcal{K}_{2}\left(\frac{1}{z}\right)}-\frac{\mathcal{K}_{3}\left(\frac{1}{z}\right)}{2 z \mathcal{K}_{2}\left(\frac{1}{z}\right)}\right)\right] \\
& +\tau v^{4} f^{(0)} \frac{p_{, \mu} h^{\mu \nu}}{c \tilde{\rho}} \frac{v_{\nu}}{z c^{2}}
\end{aligned}
$$

where $\kappa_{T}$ the isothermal compressibility, $\beta$ the thermal expansion coefficient and $\tilde{\rho}=(n \varepsilon+p) / c^{2}$ where $\varepsilon$ and $p$ are the internal energy and pressure respectively which, in turn, have to still be expressed in terms of the scalar indepentend variables, 
$T$ and $n$, through some equation of state. This is required for consistency, since the chosen representation is $n, T$ and $u^{\nu}$. Then, the forces present in the solution $\phi$ are exclusively the temperature and density gradients. By means of the Enskog transport equation [8], the heat flux in the comoving frame can be shown to be

$$
J_{[Q]}^{\mu}=n\left\langle m c^{2} v^{\mu}\right\rangle=m c^{2} \int v^{\mu} f^{(0)} \phi \gamma d v^{*}
$$

such that, since $f^{(0)} \phi$ is coupled with $\nabla T$ and $\nabla n$, the corresponding constitutive equation has the following form

$$
J_{[Q]}^{\mu}=-h_{\nu}^{\mu}\left(L_{T T} T^{, \nu}+L_{n T} n^{, \nu}\right)
$$

where $L_{T T}$ is an "effective relativistic thermal conductivity". The new transport coefficient $L_{n T}$ has no classical counterpart. We would like to remark that equations similar in structure to Eq. (15) were proposed by previous authors [20, 22, 23] but also to strongly emphasize that the term proportional to the gradient of $n$ arises here since the pressure $p$ is not an independent variable, and thus the rules of linear irreversible thermodynamics compels us to express $\nabla p$ in terms of $\nabla n$ and $\nabla T$.

The fact that kinetic theory predicts a heat flux that depends only on gradients of state variables is widely accepted. However, as mentioned above, the phenomenology seems to relate it with the acceleration. This ambiguety remains an open issue that will be addressed elsewhere. In the rest of this work we focus on the kinetic theory results given by Eq. (15) or variations of it [20, 22, 23].

\section{RELATIVISTIC IRREVERSIBLE THERMODYNAMICS: CAUSALITY AND STABILITY}

As a natural extension of the arguments summarized in Sect. II, it is intuitively clear that the formulation of relativistic irreversible thermodynamics should start 
by considering systems whose states are described through field variables which are continuous functions of the space-time coordinates $x_{\alpha}=\left(x_{1}, x_{2}, x_{3}, c t\right)$ and will hence satisfy conservation equations. If $N^{\alpha}=n u^{\alpha}$ is the particle flux,

$$
N_{; \alpha}^{\alpha}=0
$$

is the statement of conservation of particles, i. e. the continuity equation. For the other two state variables, $u^{\nu}$ and $\varepsilon$ (or $T$ ) the balance equations are no longer independent. Moreover, the resulting equations must imply the local relativistic versions of the first and second laws of thermodynamics. The energy-momentum conservation law

$$
T_{; \nu}^{\mu \nu}=0
$$

can be obtained directly from Boltzmann's equation. However, the still open question is whether this is to be matched with the conservation law implyied by Einstein's field equation

$$
R_{\mu \nu}-\frac{1}{2} R g_{\mu \nu}=\kappa \mathcal{T}_{\mu \nu}
$$

where $R_{\mu \nu}-\frac{1}{2} R g_{\mu \nu}$ is Einstein's tensor describing the geometry of space-time. In Eq. (18), $\mathcal{T}_{\mu \nu}$ is the energy-momentum tensor which accounts for the properties of matter and $\kappa$ is the coupling constant. Since the covariant derivative of the Einstein tensor vanishes one has a conservation law $\mathcal{T}_{; \nu}^{\mu \nu}=0$. As already shown by Einstein himself [24], the Euler equations of relativistic hydrodynamics for an inviscid fluid follow directly when

$$
\mathcal{T}^{\mu \nu}=\frac{n \varepsilon}{c^{2}} u^{\mu} u^{\nu}+p h^{\mu \nu}
$$

However, he never address the dissipative case.

On the other hand, from a purely hydrodynamic point of view and in order to include heat in the total energy account, Eckart resorted to an irreducible decomposition for $T^{\mu \nu}$ and identified relativistic corrective terms proportional to the heat 
flux. His proposal, in his own words, was that no assumptions would be made for the form of $T_{\mu \nu}$, but it would be used to define other quantities such as the internal energy $\varepsilon$ and the heat flux $J_{[Q]}^{\mu}$. He thus obtained

$$
T^{\mu \nu}=\frac{n \varepsilon}{c^{2}} u^{\mu} u^{\nu}+p h^{\mu \nu}+\Pi^{\mu \nu}+\frac{1}{c^{2}}\left(J_{[Q]}^{\mu} u^{\nu}+J_{[Q]}^{\nu} u^{\mu}\right)
$$

although he never mentioned whether Eq. (20) should be the one compatible with Eq. (18). One should be cautious in undertaking this step since heat is not a state variable, but energy in transit. An alternative to this proposal was examined in Ref. [25] following Meixner's ideas by omitting the last two terms in Eq. (20) and introducing heat in the definition of a total energy flux in a similar fashion as done in non-relativistic LIT. This approach led to a debate regarding the question of whether the heat should be included in Einstein's field equation through the stress-energy tensor [26] [27], .

In this work as in Ref. [8] and Section IV of Ref. [28], the tensor proposed by Eckart is used while Meixner's formalism is kept as a separate alternative. Indeed, introducing the constitutive equation (15) in the set obtained from (16), (17) and (201) a closed set of equations is obtained which will not be repeated here. The next step in order to examine if Onsager's linear regression of fluctuations hypothesis holds true implies linearizing such system by setting $T=T_{0}+\delta T, n=n_{0}+\delta n$ and $u^{k}=\delta u^{k}(k=1,2,3)$ since in equilibrium and in the comoving frame $u_{0}^{k}=0$. Here the naught subscript denotes equilibrium values and $\delta$ prefix the fluctuations around them. The linearized set of equations, which the fluctuations should follow according to the linear regression assumption, is given by

$$
\begin{gathered}
\delta \dot{n}+n_{0} \delta \theta=0 \\
\frac{1}{c^{2}}\left(n_{0} \varepsilon_{0}+p_{0}\right) \delta \dot{u}_{\nu}+\frac{1}{\kappa_{T}} \delta n_{, \nu}+\frac{1}{\beta \kappa_{T}} \delta T_{, \nu}-2 \eta\left(\delta \tau_{\nu}^{\mu}\right)_{; \mu}-\zeta \delta \theta_{, \nu}-\frac{1}{c^{2}}\left(L_{T T} \delta \dot{T}_{, \nu}+\dot{F}_{\nu}\right)=0
\end{gathered}
$$




$$
n C_{n} \delta \dot{T}+\frac{\beta T_{0}}{\kappa_{T}} \delta \theta-\left[h_{\nu}^{\mu}\left(L_{T T} T^{, \nu}+F^{\nu}\right)\right]_{; k}=0
$$

To arrive at this set of equations the local equilibrium assuption has been used to express $\varepsilon=\varepsilon(n, T)$ and $p=p(n, T) . C_{n}$ is the specific heat at constant particle number density. We conveniently introduced a vector quantity $F^{\nu}$ as a wildcard which is associated with the hydrodynamic acceleration in Eckart's fomalism, with a gradient of number density in the one proposed in Ref. [8], or some other gradient following Israel's early work [23] or the calculations shown in relativistic kinetic theory standard books [20, 22]. As was emphatically pointed out in previous work [6], the exponential growth of perturbations can be picked up simply by performing a Fourier-Laplace transform followed by the calculation of the curl of Eq. (22). This isolates the transverse mode and allows for the assesement of its behavior in time. If $F^{\nu}$ is associated with a time derivative in the velocity, the procedure yields a cuadratic equation for the Laplace variable with one postive root which in turn leads to the unphysical growth of fluctuations. However, if $F^{\nu}$ is indeed a gradient of a thermodynamic quantity, as predicted by kinetic theory, its curl vanishes and a first order equation is obtanied. This yields an exponential decay for the transverse fluctuations with which one concludes that the instability of the equilibrium state is not longer present. For the longitudinal mode one obtains

$$
\begin{gathered}
\tilde{\rho}_{0} \delta \dot{\theta}+\frac{1}{n \kappa_{T}} \nabla^{2} \delta n+\frac{\beta}{\kappa_{T}} \nabla^{2} \delta T \\
-A \nabla^{2} \delta \theta-\frac{L_{T T}}{c^{2}} \nabla^{2} \delta \dot{T}-\frac{1}{c^{2}} \dot{F}_{; \nu}^{\nu}=0
\end{gathered}
$$

Equations (21), (23) and (24) constitute a set of three coupled equations for $\delta T$, $\delta n$ and $\delta \theta$. Taking their Fourier-Laplace transform a set of algebraic equations is obtained which may be solved to yield a dispersion relation from which its roots may be obtained. After a rather long calculation by inverse transformation one obtains 
explicit solutions for $\delta n(\vec{r}, t)$ and $\delta T(\vec{r}, t)$.

As it is well known the normalized autocorrelation function of the density fluctuations is proportional to the dynamic structure factor [17, 18, 29]. This calculation, in the non-relativistic case, yields the well known Rayleigh-Brillouin spectrum predicted by Landau and Placzek in 1934 [30] and measured experimentally for Argon in 1966 by Boon et al. [17]. This is one of the many experimental confirmations of the linear regression assumption.

What we expect to find in the relativistic case is precisely a relativistic correction to this spectrum. This does not occur when if $F^{\mu} \propto \dot{u}^{\mu}$. As shown in Ref. [28], the spectrum simply does nor exist. On the other hand, if $F^{\mu} \propto h^{\mu \nu} n_{, \nu}$ we do indeed recover the spectrum with relativistic corrections to Rayleigh's peak, which in the non-relativistic limit reduces to the classical form. Even if the experiment is not or cannot be performed for technological reasons, the result speaks in favor of a constitutive equation in the form given in Refs. [8, 20, 22, 23] and not Eckart's one. It is precisely due to the structure of Eq. (15) that one can show that the resulting linearized relativistic hydrodynamic equations are in full agreement with Onsager's linear regression assumption [1] [9]. This in turn implies that the equilibrium state is thermodynamically stable.

\section{DISCUSSION}

In the previous sections, we have reviewed recent results in relativistic LIT and kinetic theory and pointed out some key theoretical arguments related to them. In

Sect. II, we emphasized the meaning of the generic instabilities found in Ref. [7] as violations of Onsager's linear regression assumption. This hypothesis, as dicussed above, lies deep inside the theoretical setup of LIT and its violation is reason enough to discard a theory of irreversible processes. Based on this violation by Eckart's 
linear theory, higher order formalisms were developed and now generally favored even though they also have been objected [31, 32]. However, based in our recent results and as outlined here in Sects. III and IV, the source of this unphysical behavior is the coupling of heat with acceleration assumed by Eckart's theory which is in disagreement with standard kinetic theory. These facts do not rule out the possibility of considering higher order, or extended, theories [33 35] but questions the need for them. That is, the unphysical behavior found in Eckart's formalism is corrected once kinetic theory is used in order to construct a heat flux tensor. These findings reopen the possibility of modeling relativistic fluids with first order theories.

In Sect. II we outlined the calculation of Ref. [8] where we obtained a constitutive equation that couples the heat flux with the thermodynamic forces $\nabla T$ and $\nabla n$. This is clearly consistent with the generalization of Onsager's theory since it couples fluxes with thermodynamic forces of the same tensorial rank, the former in this case being the heat flux and the latter $h^{\mu \nu} T_{, \nu}$ and $h^{\mu \nu} n_{, \nu}$ in the $\left(n, T, u^{\mu}\right)$ representation. This constitutive equation is then introduced in Sect. IV to argue that with it no stability nor causality issues arise in the relativistic transport equations. The details of these calculations can be found in Refs. [6] and [28]. We also comment on the causality and refer the reader to a recent publication in this subject [36].

This work also serves to update a discussion that has been going on for some time. The first issue concerns the nature of the time component of heat flux four-vector. It is important to notice that we are using a projector in the constitutive equation (15). This was questioned in previous work [25] but has since been analyzed. Indeed, in Ref. [21] we show that a generalized relativistic Chapman-Enskog procedure for the relativistic Boltzmann equation is possible and consistent with irreversible thermodynamics. We concluded that the fourth component of the heat flux as predicted by such a formal approach is still the internal energy itself. Thus, no "disspation in the time direction" is present in the theory and the projector should be included in 
the constitutive equation as done by most authors.

Secondly, the presence of heat in the stress-energy tensor must be addressed here following the discussion in Refs. [26] and [27]. In Sect. IV, in particular in Eq. (20), we clearly state that here as in some of our recent work, the stress-energy tensor considered is indeed the one proposed by Eckart strictly from a mathematical argument. However it still lacks a kinetic justification in the laboratory frame, eventhough some work along this line can be found in Ref. [37]. Nevertheless the relativistic heat terms can be readily shown to be present in the tensor in the comoving frame where most of our calculations are performed. Indeed we have lately adopted such a tensor as the fluid's stress energy tensor where, as mentioned earlier, the heat flux is included and the first term corresponds to an internal energy flux. Both energy and momentum balances are obtained from the general conservation law $T_{; \nu}^{\mu \nu}=0$ as pointed out above. On the other hand, the work in Ref. [25], which raised the discussion leading to Refs. [26] and [27], used a Meixner-like approach where the heat is not included in the stress energy tensor but is introduced in the system via the construction of a total energy flux. The predictions found in [28] finally lead to the criteria which, on experimental grounds, should prevail to decide which of the approaches is the correct one: Eckart's [5], the one proposed by Sandoval-Villalbazo and Garcia-Colin in Ref. [25], or the one in Ref. [8]. This is still an open question.

It has to be clarifyed here that in the present work as well as in Refs. [8] and Section IV of Ref. [28], the tensor given in Eq. (20) is used as the fluid's stress energy tensor eventhough a kinetic proof of its structure, other than in the commoving frame, is still to be established. We consider that the question of whether this same tensor is the one to be included in Einstein's field equation is still open and should be addressed. The actual solution of such equation including heat sources should be critically analyzed in order to asses the possible effects of including such terms. This is clearly outside the scope of this review and will be addressed elsewhere. 
The authors deeply acknowledge the valuable comments and suggestions of Prof. L.S. Garcia-Colin.

[1] L. Onsager, Phys. Rev. 37, 405 (1931); ibid 38, 2265 (1931).

[2] L. Onsager and S. Machlup, Phys. Rev. 91, 1505 (1953), ibid 91, 1512 (1953).

[3] H.B.G. Casimir, Rev. Mod. Phys. 17, 343 (1945).

[4] J. Meixner, Ann. Physik 39, 333 (1941); ibid 41, 409 (1942); ibid 43, 244 (1943); Zeit. Phys. Chem. B53, 235 (1993); Zeits. Phys 124, 129 (1944); Zeits. Naturf. 4A, 594 (1949).

[5] C. Eckart, Phys. Rev. 58, 267 (1940); ibid 58, 919 (1940).

[6] A.L Garcia-Perciante, L. S. Garcia-Colin and A. Sandoval-Villalbazo, Gen. Rel. Grav. 411645 (2009).

[7] W. A. Hiscock and L. Lindblom, Phys. Rev. D 31, 725 (1985).

[8] A. Sandoval-Villalbazo, A. L. Garcia-Perciante and L. S. Garcia-Colin, Physica A 388 3765 (2009).

[9] S.R. de Groot and P. Mazur, Non equilibrium thermodynamics, Dover publications Inc. Mineola N.Y. (1984).

[10] L.S. Garcia-Colin and F.J. Uribe, J. Non-equlib. Thermodyn. 16, 89 (1991).

[11] S.R. de Groot, The thermodynamics of irreversible processes, North Holland publishers company, Amsterdam (1952).

[12] L.E. Reichl, A modern course in statistical physics 2nd Ed., University of Texas Press, Austin (1998) .

[13] J. Keizer, Statistical mechanics of non-equilibrium processes, Springer-Verlag. N.Y. (1986).

[14] H.B. Callen, Thermodynamics and an introduction to thermostatics 2nd Ed., John 
Wiley and Sons, N.Y. (1985).

[15] S. Chandrasekhar, Rev. Mod. Phys. 15, 1 (1943). Reprinted in Noise and stochastic processes" by N. Wax, Dover publications Inc. Mineola N.Y. (1954) .

[16] A. Westgren, Arkiv for Mathematik, Astronomi och Fysik 11 numbers 8,14 (1916) and 13,14 (1918).

[17] J.P. Boon and S. Yip, Molecular Hydrodynamics, Dover publications Inc. Mineola N.Y. (1991).

[18] B.J. Berne and R. Pecora; Dynamic light scattering and its applications to Physics, Chemistry and Biology, Wiley-Interscience, N.Y. (1976)

[19] W. H. Cropper; Am. J. Phys. 54, 1068 (1986).

[20] C. Cercignani and G. Medeiros Kremer; The Relativistic Boltzmann Equation: Theory and Applications, Birkhauser, Berlin (2002).

[21] A.L Garcia-Perciante, A. Sandoval-Villalbazo and L. S. Garcia-Colin; Physica A 387, 5073-5079 (2008).

[22] S. R. de Groot, W. A. van Leeuwen and Ch. van der Wert; Relativistic Kinetic Theort, North Holland Publ. Co., Amsterdam (1980).

[23] W. Israel; J. Math. Phys 4, 1163 (1963).

[24] A. Einstein, The meaning of relativity, Princeton University Press, Princeton (2005).

[25] L. S. Garcia-Colin and A. Sandoval-Villalbazo; J. Non-equib. Thermodyn. 31, 11 (2006).

[26] W. Muschik and H. H. von Borzeszkowsky; J. Non-equib. Thermodyn. 32, 181 (2007).

[27] L. S. Garcia-Colin and A. Sandoval-Villalbazo; J. Non-equib. Thermodyn. 32, 187 (2007).

[28] A.L Garcia-Perciante, L. S. Garcia-Colin and A. Sandoval-Villalbazo; Phys. Rev. E 79 066310 (2009).

[29] R. Mountain; Rev. Mod. Phys. 38, 205 (1966). 
[30] L. Landau and G. Placzek; Physik Z. Sowjetunion 5, 172 (1934).

[31] L. S. Garcia-Colin; Rev. Mex. Fis. (in english) 34, 344 (1988).

[32] L. S. Garcia-Colin; Mol. Phys. 86, 697 (1995).

[33] W. Israel; Ann. Phys. (N. Y.) 100, 310 (1976).

[34] W. Israel and J. M. Stewart; Ann. Phys. (N. Y.) 118, 341 (1979).

[35] D. Jou, J. Casas-Vazquez and G. Lebon; Extended Irreversible thermodynamics, 2nd ed. springer-Verlag, Berlin (1997).

[36] A.L Garcia-Perciante, A. Sandoval-Villalbazo; Festschrift in honor of Prof. Leopoldo Garcia-Colin Scherer (in press), arXiv:1001.4832.

[37] A. R. Sagaceta-Mejia, A.L Garcia-Perciante and A. Sandoval-Villalbazo; arXiv:0912.4369. 